\title{
On The Performance of Underlay Relay Cognitive Networks
}

\author{
Khuong Ho-Van ${ }^{1}$, Vo Nguyen Quoc Bao ${ }^{2}$ \\ 1 Telecommunications Engineering Department, Ho Chi Minh City University of Technology, Ho Chi \\ Minh City, Vietnam \\ ${ }^{2}$ School of Telecommunications, Posts and Telecommunications Institute of Technology, Ho Chi Minh \\ City, Vietnam
}

Correspondence: Khuong Ho-Van, khuong.hovan@yahoo.ca

Manuscript communication: received 14 July 2013, accepted 12 August 2013

\begin{abstract}
The bit error rate (BER) performance of underlay relay cognitive networks in the presence of Rayleigh fading is thoroughly analyzed in this paper. New exact and asymptotic analytic expressions under consideration of both interference power constraint and maximum transmit power constraint are derived in closed-form and are extensively corroborated by Monte-Carlo simulations. These expressions facilitate in evaluating effectively the network performance behaviour in key operation parameters as well as in optimizing system parameters. A multitude of analytical results expose that underlay relay cognitive networks experience the performance saturation phenomena while their performance considerably depends on the number of hops for the linear network model. Additionally, optimum relay position is significantly dependent of maximum transmit power, maximum interference power, and licensee location. Moreover, the appropriate order of locating unlicensees with different maximum transmit power levels can dramatically improve the network performance.
\end{abstract}

Keywords- Cooperative communications, underlay cognitive radio network, Rayleigh fading, bit error rate.

\section{INTRODUCTION}

Currently there are two motivations to urge devising new technologies for high spectrum utilization efficiency. On the one hand, the current spectrum utilization efficiency is rather low, as reported in an extensive survey on spectrum utilization carried out by the Federal Communications Commission [1]. On the other hand, new wireless applications undoubtedly require wide transmission bandwidth but must compete each other for limited available spectrum. The solution to the popularly reminded problem of spectrum underutilization is the cognitive radio technology [2]. Integrated with this technology, un-licensees can still operate in the frequency band, which has been primarily allotted to licensees, without causing any harm to licensees. As a result, the corresponding spectrum utilization can be substantially increased. In general, it has been largely shown that in cognitive radio-based communications un-licensees can work in three modes: interweave; overlay; and underlay [3].

This paper investigates the underlay mode that is widely accepted for its rather low implementation complexity [3]. In this mode, un-licensees must adaptively control their transmit power so that the induced interference is maintained within levels which can be tolerated by the licensees. Therefore, the transmission range of un-licensees is drastically shortened. However, it is evident that integrated with multi-hop communication techniques which exploit shorter range communication for lower path loss, underlay multi-hop cognitive networks can efficiently overcome the above drawback. In the multi-hop communications, information from a source is relayed to a destination through several relays on the hop-by-hop basis in the decodeand-forward (DF) and amplify-and-forward (AF) manner [4]. In the former, the relay decodes the received signal and then re-encodes the decoded information before relaying through the next hop. In the latter, the relay just amplifies the received signal and forwards the resulting signal through the next hop. The DF protocol is considered in the present work.

Several works study the outage probability of underlay $\mathrm{DF}^{1}$ multi-hop cognitive networks in [3,5-11]. More specifically, the outage probability expression for twohop communications was derived in [3,5-10] while the approximate outage probability expression for multihop cognitive networks was proposed in [11]. Additionally, the Rayleigh fading was assumed in the analyses in $[3,6-9,11]$ while the more flexible Nakagami- $m$ fading model [12] was considered in [5, 10]. In the same context, the authors in [13-25] analyze the outage probability of underlay DF cooperative cognitive networks. It is noted that the outage probability analysis can provide an insight into the information-theoretic performance limit and motivate code design to reach it. However, effective determination of this limit is rather problematic. Meanwhile, the BER analysis shows the real -not a limit- system performance for a given target spectral efficiency (i.e., modulation level) and hence, is of greater practical importance. Nevertheless, in spite

\footnotetext{
${ }^{1}$ This paper concentrates on the DF protocol, and hence, the literature survey on underlay AF cooperative/multi-hop cognitive networks is not necessary.
} 
of the undoubted usefulness of underlay DF multihop cognitive networks, only limited investigations on the BER analysis of these systems have been reported in the open technical literature ${ }^{2}$ [27-29]. In addition, the works in [27, 28] do not investigate the maximum transmit power constraint on unlicensees, which is strictly required in cognitive radio-based networks. Likewise, the work in [29] provides only an approximate closedform expression for the BER of two-hop cognitive networks.

Motivated by the above, the main contribution of this paper is the analytic performance evaluation of the bit error rate of such networks. More specifically, a novel exact closed-form expression is derived for the BER of underlay DF multi-hop cognitive networks with arbitrary number of hops in the presence of Rayleigh fading. Both the interference power constraint and the maximum transmit power constraint are taken into account. Additionally, the corresponding asymptotic performance for large maximum transmit power (i.e., relaxing the maximum transmit power constraint) or large maximum interference power (i.e., ignoring the interference power constraint) is also analysed. Notably, the BER of conventional DF multi-hop networks in [30] is a special case of our asymptotic results for large maximum interference power while our asymptotic results for large maximum transmit power can be used to compute the BER of underlay DF multihop cognitive networks under only interference power constraint such as $[7,13]$. All derived expressions are verified extensively through comparisons with results from computer simulations. Interestingly, it is clearly shown that underlay DF multi-hop cognitive networks considerably suffer error floor phenomenon while the associated BER performance depends significantly, as expected, on the number of hops for the linear network topology. Moreover, optimum relay position is dramatically dependent of maximum transmit power, maximum interference power, and licensee location. Furthermore, the appropriate order of positioning unlicensees with different maximum transmit powers can substantially impact the system performance.

The structure of this paper is as follows: Section 2 presents the system model. The error probability of underlay DF multi-hop cognitive networks is exactly analyzed in Section 3 while the asymptotic analysis is discussed in Section 4. Simulated and analytical results for the corresponding performance assessment are presented in Section 5. Finally, Section 6 provides closing remarks.

\section{System Model}

The underlay $N$-hop cognitive network under investigation is depicted in Figure 1. The transmission in the unlicensed network starts from $U_{0}$ and ends up

\footnotetext{
${ }^{2}$ Recently, the authors in [26] studied the approximate symbol error rate of underlay $A F$ multi-hop cognitive networks with only interference power constraint at the unlicensed source but both power constraints at the unlicensed relay.
}

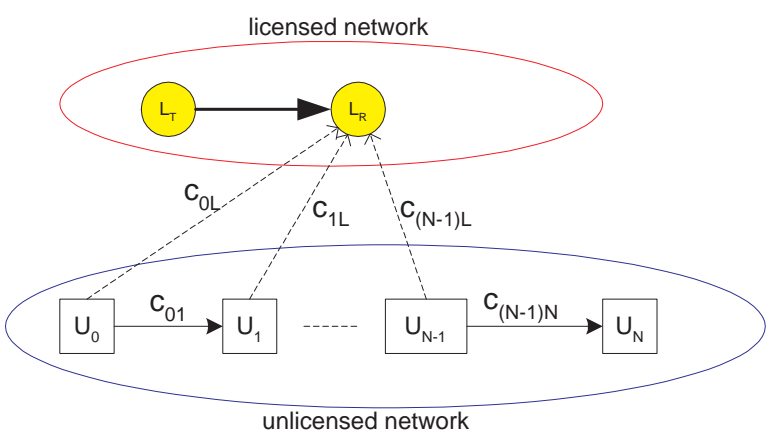

Figure 1. Network model. $L_{T}$ and $L_{R}$ denote the transmitter and the receiver in the licensed network, respectively.

at $U_{N}$ through $(N-1)$ intermediate unlicensed users $\left\{U_{1}, \ldots, U_{N-1}\right\}$, interfering with the licensed users, $L_{R}$. We assume non-identical independent frequency-flat fading which follows the Rayleigh distribution. To this end, the wireless channel coefficient between the transmitter $U_{a}$ and the receiver $U_{b}$ is ${ }^{3} c_{a b} \sim \mathcal{C N}\left(0, \frac{1}{\varrho_{a b}}=\right.$ $d_{a b}^{-\tau}$ ) where $d_{a b}$ and $\tau$ denote the distance between two users and the involved path-loss exponent, respectively, while $a \in\{0,1, \ldots, N-1\}$ and $b \in\{1,2, \ldots, N, L\}$, with $L$ denoting the index of licensed user $L_{R}$ [31].

It is also recalled that $N$-hop wireless transmission takes place in $N$ phases. In more details, in the $n$th phase, the transmitter $U_{a}$ broadcasts a sequence of $q$ modulated symbols $\mathbf{y}_{a}=\left[y_{a}(1), y_{a}(2), \ldots, y_{a}(q)\right]$, each with symbol energy, $\mathcal{P}_{a}=\mathcal{E}\left\{\left|y_{a}(u)\right|^{2}\right\}$, where $\mathcal{E}\{\cdot\}$ and $u$ denote the expectation and time index, respectively. Based on this, this symbol sequence is demodulated by the receiver $U_{n}$ and re-modulated as $\mathbf{y}_{n}=$ $\left[y_{n}(1), y_{n}(2), \ldots, y_{n}(q)\right]$, each with the symbol energy, $\mathcal{P}_{n}$. Then, they are forwarded to the unlicensed user $U_{n+1}$ during the $(n+1)$ th phase ${ }^{4}$. Based on this, the received signal in the $n$th phase can be modelled as follows ${ }^{5}$ :

$$
z_{a n}=c_{a n} y_{a}+x_{a n}
$$

where $z_{a n}$ is the signal received at the user $U_{n}$ from the user $U_{a}$ with $a=n-1$ and $x_{a n} \sim \mathcal{C N}\left(0, \mathcal{N}_{n}\right)$ being the additive noise at the user $U_{n}$. It should be noted that more practically, $x_{a n}$ at the unlicensed receiver consists of two components: $i$ ) the actual noise at the receiver, and $i i)$ the interference from the licensed transmitters, $[7-9,19,20,23]$. According to the central limit theorem [32], the interference component becomes Gaussian distributed as long as the number of licensed interferers is large enough. In underlay cognitive radio networks, unlicensed users operate in the opportunistic manner and hence, they may be interfered by a large number of licensed users. As such, the assumption of Gaussian-distributed interference from licensed users can be valid in several practical scenarios and is widely

\footnotetext{
${ }^{3} \mathrm{C} \sim \mathcal{C N}(\mathcal{M}, \mathcal{V})$ denoting a circular symmetric complex Gaussian random variable with mean $\mathcal{M}$ and variance $\mathcal{V}$.

${ }^{4}$ For the sake of notation simplicity and to avoid any confusion, the time index is henceforth omitted.

${ }^{5}$ This received signal model is also valid for the communication between the unlicensed user and the licensed user.
} 
accepted/exploited in most recent research works $[3,5$, $6,11,13-18,21,22,24-29]$. Under this assumption, $\mathcal{N}_{n}$ is considered as the total variance of the noise at the unlicensed user and the interference from the licensed transmitters.

It is noted here that operating in the underlay mode, as in e.g., [3], the unlicensed user $U_{a}$ must set its transmit power according to $\mathcal{P}_{a}=\min \left(\mathcal{I}_{m} /\left|c_{a L}\right|^{2}, \mathcal{P}_{a m}\right)$. This holds for the maximum transmission range and for meeting both the corresponding interference power constraint, $\mathcal{P}_{a} \leq \mathcal{I}_{m} /\left|c_{a L}\right|^{2}$, and maximum transmit power constraint, $\mathcal{P}_{a} \leq \mathcal{P}_{a m}$. Here, $\mathcal{I}_{m}$ is the maximum interference power that the licensed user can tolerate and $\mathcal{P}_{a m}$ is the maximum transmit power designed for unlicensed users. In this paper, different unlicensed users allocated with different maximum interference power levels is investigated and hence, more general than all previous works with the assumption of the same maximum interference power level for all unlicensed users such as [3, 5, 6, 8-11, 14-29]. As analysed thoroughly in $[3,5,6,11,14,15,18,24-26,29], \mathcal{I}_{m}$ stands implicitly for the interference limit from unlicensed users and excludes interference generated by licensed users. Equivalently, the licensed networks are implicitly assumed to operate reliably for interference levels caused by unlicensed users up to $\mathcal{I}_{m}$, regardless of the interference already existing in these networks. In other words, licensee-to-licensee interference has not been necessarily considered when formulating $\mathcal{P}_{a}$. Also, in order to set the transmit power of unlicensees, the channel coefficient $c_{a L}$ must be available at the unlicensees. Obtaining channel state information (CSI) at a certain level of accuracy is almost feasible and elaborately discussed in most works on the cognitive radio technology, e.g. [3, 23], and hence, more investigation on the channel estimation issue is not really necessary. In the sequel, we assume that perfect CSI is available. Based on this transmit power setting, (1) results in the following instantaneous signal-to-noise ratio (SNR) in the $n$th phase:

$$
\mu_{a n}=\frac{\mathcal{P}_{a}\left|c_{a n}\right|^{2}}{\mathcal{N}_{n}}=\min \left(\frac{\mathcal{I}_{m}}{\left|\mathcal{c}_{a L}\right|^{2}}, \mathcal{P}_{a m}\right) \frac{\left|c_{a n}\right|^{2}}{\mathcal{N}_{n}} .
$$

The cumulative distribution function (cdf) of $\mu_{a n}$ is obtained by recalling that the cdf of $\eta_{a n}=$ $\min \left(\mathcal{I}_{m} /\left|c_{a L}\right|^{2}, \mathcal{P}_{a m}\right)\left|c_{a n}\right|^{2}$ is expressed as follows [3, eq. (8)]:

$$
F_{\eta_{a n}}(x)=\left(\frac{x e^{-\frac{\epsilon_{a n \rho a n} \mathcal{I}_{m}}{\mathcal{P}_{a m}}}}{x+\epsilon_{a n} \mathcal{I}_{m}}-1\right) e^{-\frac{\varrho_{a n} x}{\mathcal{P a m}}}+1,
$$

where $\epsilon_{a n}=\varrho_{a L} / \varrho_{a n}$. Given that $\mu_{a n}=\eta_{a n} / \mathcal{N}_{n}$, it immediately follows that the cdf of $\mu_{a n}$ can be expressed as

$$
\begin{aligned}
F_{\mu_{a n}}(x) & =\operatorname{Pr}\left\{\mu_{a n} \leq x\right\} \\
& =\operatorname{Pr}\left\{\frac{\eta_{a n}}{\mathcal{N}_{n}} \leq x\right\} \\
& =F_{\eta_{a n}}\left(\mathcal{N}_{n} x\right) .
\end{aligned}
$$

The corresponding probability density function (pdf) is derived by differentiating (4) with respect to $x$, namely, $f_{\mu_{a n}}(x)=d F_{\mu_{a n}}(x) / d x$. This yields the closed-form expression as (5).

\section{Exact Error Probability Analysis}

The corresponding error rate analysis can be completed in the following two steps:

- Step 1: Computation of the accurate closed-form average $\mathrm{BER}, B_{\mathcal{e}}(n)$, in the $n$th phase.

- Step 2: Use of $B_{e}(n)$ for $n=1,2, \ldots, N$ in Step 1 to calculate the average BER of the underlay multihop cognitive networks with the aid of [30, eq. (9)], namely,

$$
B_{e}=\sum_{n=1}^{N}\left[B_{e}(n) \prod_{j=n+1}^{N}\left(1-2 B_{e}(j)\right)\right] .
$$

It is noted that the average end-to-end BER in (6) takes into account the error propagation in the multihop communications which happens when a bit is erroneously decoded on one hop but still forwarded to another hop. Also, Step 1 is undoubtedly the most critical step. The average BER in the $n$th phase is computed with the aid of $f_{\mu_{a n}}(x)$ and the corresponding instantaneous BER. More specifically, given the instantaneous BERs for square $M$-ary Quadrature Amplitude Modulation ( $M-\mathrm{QAM})$ with $M=2^{h}$ ( $h$ even) and rectangular $M-Q A M$ with $M=2^{h}(h$ odd) in [33, eq. (16)] and $\left[33\right.$, eq. (22)], respectively ${ }^{6}$, the $B_{e}(n)$ can be expressed as $(7)$ where $\Lambda(s, v, M ; x)$ is given in (8) and

$$
\begin{aligned}
p & =\frac{3}{M-1}, \\
t & =\frac{6}{W^{2}+R^{2}-2^{\prime}} \\
W & =2^{(h-1) / 2}, \\
R & =2^{(h+1) / 2} .
\end{aligned}
$$

Furthermore, the notations $\lfloor$.$\rfloor and Q($.$) are the$ floor function and the one dimensional Gaussian $Q$ function [12], respectively, which are both included as standard built-in functions in popular mathematical software packages such as MAPLE and MATLAB. Evidently, the derivation of a closed-form expression for $B_{\mathcal{e}}(n)$ is subject to analytical evaluation of the two infinite integrals in (7). To this end, by substituting (8) into (7) one obtains the following closed-form expression for the BER in the $n$th phase as (13) where $\Phi\left(s, v, M, G_{a n}, \rho_{a n}\right)$ is given in (16) and

$$
\begin{aligned}
\rho_{a n} & =\frac{\varrho_{a n} \mathcal{N}_{n}}{\mathcal{P}_{a m}}, \\
G_{a n} & =\frac{\epsilon_{a n} \mathcal{I}_{m}}{\mathcal{N}_{n}} .
\end{aligned}
$$

Moreover, the $\zeta(a, b, c)$ term in (16) is defined as:

$$
\zeta\left(\beta, G_{a n}, \rho_{a n}\right) \triangleq \int_{0}^{\infty} Q(\sqrt{\beta x}) f_{\mu_{a n}}(x) d x .
$$

\footnotetext{
${ }^{6}$ Similarly, we can derive the average BER for other modulation schemes such as M-PSK (Phase-Shift Keying).
} 


$$
f_{\mu_{a n}}(x)=\mathcal{N}_{n} e^{-\frac{\varrho_{a n} \mathcal{N}_{n} x}{\mathcal{P}_{a m}}}\left\{\frac{\epsilon_{a n} \mathcal{I}_{m} e^{-\frac{\epsilon_{a n} \mathcal{I}_{m e a n}}{\mathcal{P}_{a m}}}}{\left(\epsilon_{a n} \mathcal{I}_{m}+\mathcal{N}_{n} x\right)^{2}}+\frac{\varrho_{a n}}{\mathcal{P}_{a m}}-\frac{\varrho_{a n}}{\mathcal{P}_{a m}} \frac{\mathcal{N}_{n} x e^{-\frac{\epsilon_{a n} \mathcal{I}_{m} \operatorname{lan}}{\mathcal{P}_{a m}}}}{\varrho_{a n} \mathcal{I}_{m}+\mathcal{N}_{n} x}\right\}
$$

$$
B_{e}(n)=\left\{\begin{array}{cc}
\int_{0}^{\infty}\{\Lambda(W, t, M ; x)+\Lambda(R, t, M ; x)\} f_{\mu_{a n}}(x) d x & , h \text { odd } \\
2 \int_{0}^{\infty} \Lambda(\sqrt{M}, p, M ; x) f_{\mu_{a n}}(x) d x & , h \text { even }
\end{array}\right.
$$

$$
\Lambda(s, v, M ; x) \triangleq \frac{2}{s \log _{2} M} \sum_{k=1}^{\log _{2} s} \sum_{i=0}^{\left(1-2^{-k}\right) s-1}(-1)^{\left\lfloor\frac{i 2^{k-1}}{s}\right\rfloor}\left(2^{k-1}-\left\lfloor\frac{i 2^{k-1}}{s}+\frac{1}{2}\right\rfloor\right) Q\left(\sqrt{(2 i+1)^{2} v x}\right) .
$$

$$
B_{e}(n)=\left\{\begin{array}{cc}
\Phi\left(W, t, M, G_{a n}, \rho_{a n}\right)+\Phi\left(R, t, M, G_{a n}, \rho_{a n}\right) & , h \text { odd } \\
2 \Phi\left(\sqrt{M}, p, M, G_{a n}, \rho_{a n}\right) & , h \text { even }
\end{array}\right.
$$

$$
\Phi\left(s, v, M, G_{a n}, \rho_{a n}\right) \triangleq \frac{2}{s \log _{2} M} \sum_{k=1}^{\log _{2} s} \sum_{i=0}^{\left(1-2^{-k}\right) s-1}(-1)^{\left\lfloor\frac{i 2^{k-1}}{s}\right\rfloor}\left(2^{k-1}-\left\lfloor\frac{i 2^{k-1}}{s}+\frac{1}{2}\right\rfloor\right) \zeta\left((2 i+1)^{2} v, G_{a n}, \rho_{a n}\right) .
$$

Hence, by integrating once by parts and substituting (4), the above expression can be equivalently re-written as follows:

$$
\begin{aligned}
\zeta\left(\beta, G_{a n}, \rho_{a n}\right) & =\underbrace{\frac{1}{\sqrt{2 \pi}} \int_{0}^{\infty} F_{\mu_{a n}}\left(\frac{t^{2}}{\beta}\right) e^{-\frac{t^{2}}{2}} d t}_{\mathcal{T}_{1}} \\
& =\underbrace{\frac{1}{\sqrt{2 \pi}} \int_{0}^{\infty} e^{-\frac{t^{2}}{2}} d t}_{\mathcal{T}_{2}} \\
& -\underbrace{\frac{1}{\sqrt{2 \pi} \int_{0}^{\infty} e^{-\left(\frac{\rho_{a n}}{\beta}+\frac{1}{2}\right) t^{2}} d t}}_{\mathcal{T}_{3}}
\end{aligned}
$$

Importantly, since $Q(x) \triangleq \frac{1}{\sqrt{2 \pi}} \int_{x}^{\infty} e^{-\frac{t^{2}}{2}} d t$ it immediately follows that,

$$
\mathcal{T}_{1}=Q(0)=\frac{1}{2}
$$

Likewise, by recalling that $\int_{0}^{\infty} e^{-\frac{a t^{2}}{2}} d t \triangleq \sqrt{\frac{\pi}{2 a}}$, the following expression is straightforwardly deduced,

$$
\mathcal{T}_{2}=\frac{1}{2} \sqrt{\frac{\beta}{\beta+2 \rho_{a n}}} .
$$

Finally, the $\mathcal{T}_{3}$ integral can be solved in closed-form with the aid of [34, eq. (3.466.2)] as

$$
\begin{aligned}
\mathcal{T}_{3} & =\frac{e^{-G_{a n} \rho_{a n}}}{2} \sqrt{\frac{\beta}{\beta+2 \rho_{a n}}} \\
& -\frac{1}{2} \sqrt{2 \pi \beta G_{a n}} e^{\frac{\beta G_{a n}}{2}} Q\left(\sqrt{\left(\beta+2 \rho_{a n}\right) G_{a n}}\right) .
\end{aligned}
$$

As a result, substituting (19), (20), and (21) in (18) yields (22). Evidently, by making the necessary change of variables in (22) and substituting in (16), a closedform expression for the BER in the $n$th phase is deduced. To the best of our knowledge, (22) has not been reported in the open technical literature. It is noted here that although the derived expression is algebraically long, it is rather straightforward and convenient to handle both analytically and numerically.

\section{Asymptotic Analysis}

The asymptotic analysis for the error rate is derived by considering two extreme scenarios: i) the large maximum transmit power; ii) the large maximum interference power. Based on this:

Theorem 1 For large values of the maximum transmit power (i.e., $\mathcal{P}_{a m} \rightarrow \infty$ ), (22) reduces to

$\lim _{\mathcal{P}_{a m} \rightarrow \infty} \zeta\left(\beta, G_{a n}, \rho_{a n}\right)=\frac{1}{2}\left\{1-\frac{e^{\frac{\beta G_{a n}}{2}} Q\left(\sqrt{\beta G_{a n}}\right)}{\left(2 \pi \beta G_{a n}\right)^{-\frac{1}{2}}}\right\}$.

Proof: When $\mathcal{P}_{a m} \rightarrow \infty$, it immediately follows that $\rho_{a n} \rightarrow 0$. Then, computing $\lim _{\mathcal{P}_{a m} \rightarrow \infty} \zeta\left(\beta, G_{a n}, \rho_{a n}\right)=$ $\lim _{\rho_{a n} \rightarrow 0} \zeta\left(\beta, G_{a n}, \rho_{a n}\right)$ completes the proof. 


$$
\zeta\left(\beta, G_{a n}, \rho_{a n}\right)=\frac{1}{2}\left\{\left(e^{-G_{a n} \rho_{a n}}-1\right) \sqrt{\frac{\beta}{\beta+2 \rho_{a n}}}-\sqrt{2 \pi \beta G_{a n}} e^{\frac{\beta G_{a n}}{2}} Q\left(\sqrt{\left(\beta+2 \rho_{a n}\right) G_{a n}}\right)+1\right\} .
$$

Notably, according to $\mathcal{I}_{m}$ the value of (23) remains constant. This clearly indicates that the network suffers from the error floor for large values of $\mathcal{P}_{a m}$.

Theorem 2 For large values of the maximum interference power (i.e., $\mathcal{I}_{m} \rightarrow \infty$ ), (22) becomes

$$
\lim _{\mathcal{I}_{m} \rightarrow \infty} \zeta\left(\beta, G_{a n}, \rho_{a n}\right)=\frac{1}{2}\left\{1-\sqrt{\frac{\beta}{\beta+2 \rho_{a n}}}\right\} .
$$

Proof: The result in (24) is based on the fact that when $\mathcal{I}_{m} \rightarrow \infty$ (i.e., $G_{a n} \rightarrow \infty$ ), the second term in the bracket of (22) reduces to zero and $e^{-G_{a n} \rho_{a n}} \rightarrow 0$.

Notably, when conditioned on $\mathcal{P}_{a m}$, the value of (24) remains constant and thus, the network experiences the performance saturation phenomenon for large $\mathcal{I}_{m}$.

In summary, the asymptotic analysis is very important and provides some useful insights into the performance of underlay DF multi-hop cognitive networks as follows:

- Underlay DF multi-hop cognitive networks experience the performance saturation at either the large maximum transmit power or the large maximum interference power.

- The asymptotic analysis can be extended to include some previous works as special cases:

- Special case 1: Only interference power constraint In this paper, we consider strict transmit power constraint where both maximum transmit power constraint and interference power constraint are taken into account. Some other works such as $[7,13]$ only investigate the interference power constraint. Naturally, our asymptotic analysis is applicable to deduce the BER expression for underlay DF multihop cognitive networks under only interference power constraint. Indeed, the case of only interference power constraint corresponds to our asymptotic analysis as $\mathcal{P}_{a m} \rightarrow \infty$, and hence, the BER for this case is computed by using (23), (16), (13), and (6), sequentially. Notably, even though only interference power constraint is assumed which is possible when the unlicense is acted as a cognitive base station or a unlicensed access point with the availability of sufficient transmit power [26], the BER expression for these networks has not been reported in any publication.

- Special case 2: Traditional multi-hop systems In traditional multi-hop systems such as [30], the transmit power is just $\mathcal{P}_{a m}$. Therefore, applying our asymptotic analysis for $\mathcal{I}_{m} \rightarrow \infty$, we can infer the BER expression for traditional multi-hop systems. More specifically, this BER is computed by using (24), (16), (13), and (6), sequentially.

\section{Numerical Results}

The first part of this section provides results in Figure 2 - Figure 5. These results verify the accuracy of the derived end-to-end BER expression in (6) and the asymptotic analysis in Section 4, as well as demonstrate the performance and behaviour of underlay multi-hop cognitive networks with respect to: $i$ ) the maximum transmit power; ii) the maximum interference power; iii) the path-loss exponent; iv) the order of positioning unlicensed users with different maximum transmit power levels. Also, in this context, we investigate the 3-hop communication scenario with the coordinates of each user being arbitrarily selected as follows: $U_{0}$ at $(0,0), U_{1}$ at $(0.5,0.3), U_{2}$ at $(0.7,0.2), U_{3}$ at $(1,0)$, and the licensed user $L_{R}$ at $(0.6,0.4)$. Moreover, two typical modulation formats are considered, namely, 2-QAM for $h$ odd and 4-QAM for $h$ even. Furthermore, the noise variances at unlicensed receivers are normalized to be identical i.e., $\mathcal{N}_{n}=\mathcal{N}_{0}$ for any $n$. Without loss of generality, the results in Figure 2 - Fig. 4 are presented with the assumption that the same maximum transmit power level is applied to all unlicensed transmitters i.e., $\mathcal{P}_{0 m}=\mathcal{P}_{1 m}=\mathcal{P}_{2 m}=\mathcal{P}_{m}$. The case of different maximum transmit power levels allocated to different unlicensed transmitters is deferred to Figure 5.

Figure 2 illustrates the performance and behaviour of the underlay multi-hop cognitive network with respect to the variation of $\mathcal{P}_{m} / \mathcal{N}_{0}$ for a fixed $\mathcal{I}_{m} / \mathcal{N}_{0}$ at $15 \mathrm{~dB}$ and the path-loss exponent $\tau=3$. It is observed that the exact results in (22) match perfectly the corresponding results from computer simulations. Likewise, the offered asymptotic results in (23) are in excellent agreement with the exact results for $\mathcal{P}_{m} / \mathcal{N}_{0} \geq 13 \mathrm{~dB}$. It is also shown that underlay DF multi-hop cognitive networks reach relatively quickly the error floor and this error floor is the BER of underlay DF multi-hop cognitive networks imposed by only the interference power constraint - as discussed in Section 4. It is noted here that the error floor emerges from the fact that the transmit power of the unlicensed user is constrained by the minimum value of the maximum transmit power, $\mathcal{P}_{m}$, and the maximum interference power, $\mathcal{I}_{m}$. As a result, for the values of $\mathcal{P}_{m}$ over a certain value (e.g., about $13 \mathrm{~dB}$ in Figure 2), the corresponding transmit power is completely determined by $\mathcal{I}_{m}$, resulting in unchanged BER levels for any increase of $\mathcal{P}_{m}$. In the same context, Figure 3 investigates the BER behaviour with respect to the change of $\mathcal{I}_{m} / \mathcal{N}_{0}$ while $\mathcal{P}_{m} / \mathcal{N}_{0}$ is fixed at $15 \mathrm{~dB}$. It is clearly observed that the simulated results coincide with the corresponding simulation results, which justifies the validity of (22). Furthermore, this figure shows the performance improvement with $\mathcal{I}_{m}$ for relatively small values of $\mathcal{I}_{m}$ (e.g., $\mathcal{I}_{m} / \mathcal{N}_{0}<$ $40 \mathrm{~dB}$ ). This is rather expected since $\mathcal{I}_{m}$ upper-bounds the transmit power of unlicensed users and thus, the 


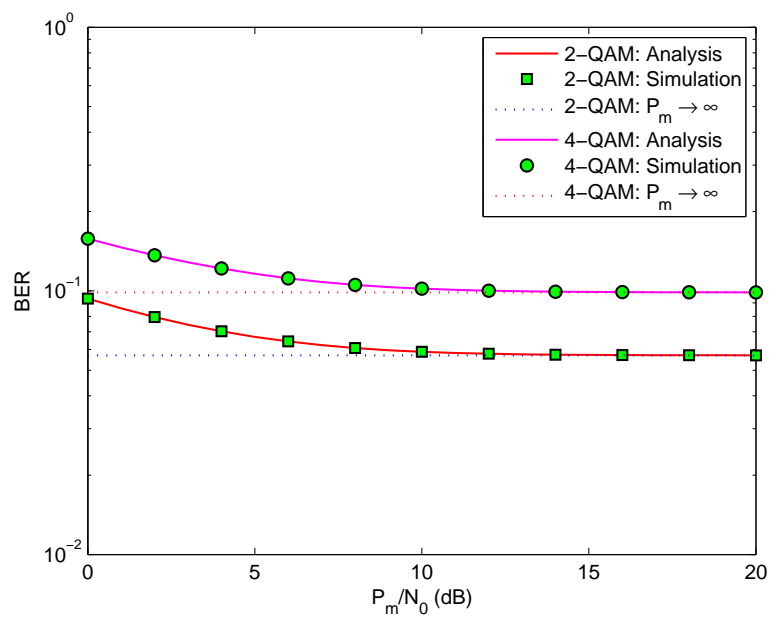

Figure 2. BER versus $\mathcal{P}_{m} / \mathcal{N}_{0}\left(\mathcal{I}_{m} / \mathcal{N}_{0}=15 \mathrm{~dB}\right)$.

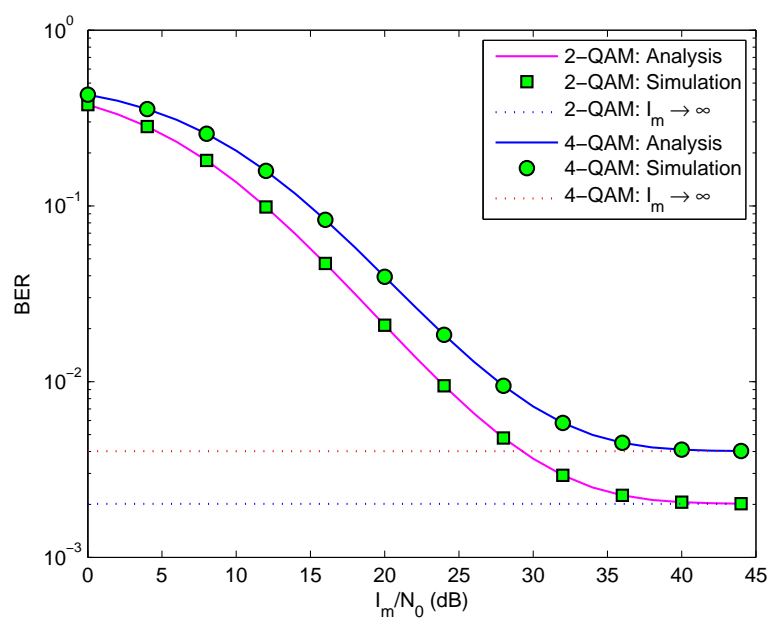

Figure 3. BER versus $\mathcal{I}_{m} / \mathcal{N}_{0}\left(\mathcal{P}_{m} / \mathcal{N}_{0}=15 \mathrm{~dB}\right)$.

higher $\mathcal{I}_{m}$ results in the higher transmit power which ultimately reduces the corresponding BER levels. For large $\mathcal{I}_{m}$ (e.g., $\mathcal{I}_{m} / \mathcal{N}_{0} \geq 40 \mathrm{~dB}$ ), the saturation in the performance is observed and this saturation level is the BER of the traditional 3-hop system as reasoned in Section 4. The interpretation of this behaviour is the same as in the depicted scenario in Figure 2. Likewise, Figure 4 illustrates the performance behaviour versus the path-loss exponent for $\mathcal{P}_{m} / \mathcal{N}_{0}=40 \mathrm{~dB}$ and $\mathcal{I}_{m} / \mathcal{N}_{0}=15 \mathrm{~dB}$. We consider typical values of the path-loss exponent $\tau=2, \ldots, 5$. The plotted results demonstrate the perfect match between analysis and simulation as well as that as $\tau$ increases, the BER performance of underlay DF multi-hop cognitive networks deteriorates significantly. As expected, this behaviour follows from the fact that the higher the path-loss, the lower the corresponding system performance. As discussed in Section 2, our BER expression is quite general, considering the scenario of different unlicensed transmitters allocated with different maximum transmit power levels. Figure 5 demonstrates its application in such a scenario. To be specific, we consider two cases with the same total maximum transmit power: i) Case 1: maximum transmit power level increases with the hop number $\left(\mathcal{P}_{0 m} / \mathcal{N}_{0}=10 \mathrm{~dB}, \mathcal{P}_{1 m} / \mathcal{N}_{0}=15 \mathrm{~dB}\right.$,

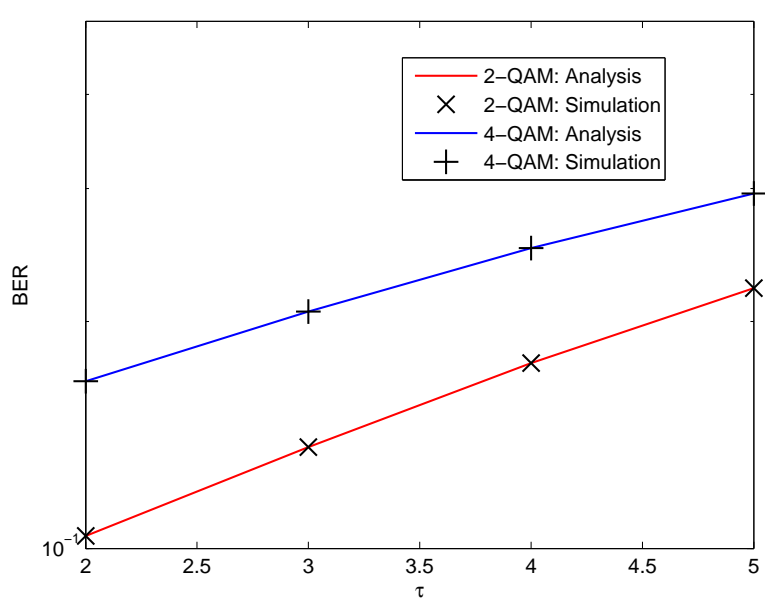

Figure 4. BER versus $\tau\left(\mathcal{P}_{m} / \mathcal{N}_{0}=40 \mathrm{~dB}, \mathcal{I}_{m} / \mathcal{N}_{0}=15 \mathrm{~dB}\right)$.

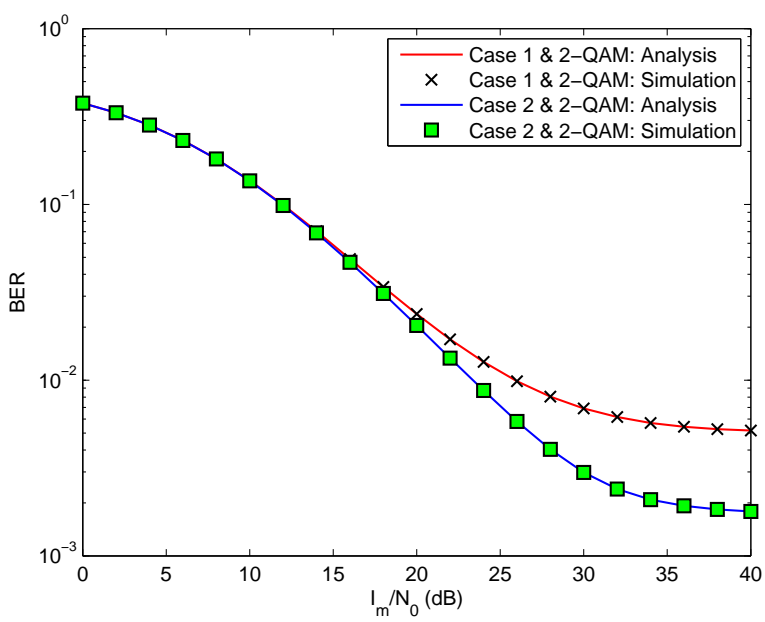

Figure 5. BER versus $\mathcal{I}_{m} / \mathcal{N}_{0}$. Case $1: \mathcal{P}_{0 m} / \mathcal{N}_{0}=10 \mathrm{~dB}, \mathcal{P}_{1 m} / \mathcal{N}_{0}=$ $15 \mathrm{~dB}, \mathcal{P}_{2 m} / \mathcal{N}_{0}=20 \mathrm{~dB} ;$ Case 2: $\mathcal{P}_{0 m} / \mathcal{N}_{0}=20 \mathrm{~dB}, \mathcal{P}_{1 m} / \mathcal{N}_{0}=15 \mathrm{~dB}$, $\mathcal{P}_{2 m} / \mathcal{N}_{0}=10 \mathrm{~dB}$.

$\mathcal{P}_{2 m} / \mathcal{N}_{0}=20 \mathrm{~dB}$ ); ii) Case 2: maximum transmit power level decreases with the hop number $\left(\mathcal{P}_{0 m} / \mathcal{N}_{0}=20 \mathrm{~dB}\right.$, $\left.\mathcal{P}_{1 m} / \mathcal{N}_{0}=15 \mathrm{~dB}, \mathcal{P}_{2 m} / \mathcal{N}_{0}=10 \mathrm{~dB}\right)$. It is shown that the analysis perfectly matches the simulation. Also, the results are reasonable in the following senses:

- In the low-to-moderate range of $\mathcal{I}_{m} / \mathcal{N}_{0}$ (e.g., $\mathcal{I}_{m} / \mathcal{N}_{0}<14 \mathrm{~dB}$ ), the BER of both cases is identical. This is because in this range, the transmit power is totally controlled by $\mathcal{I}_{m}$ and hence, the performance is independent of maximum transmit power levels.

- At large values of $\mathcal{I}_{m} / \mathcal{N}_{0}$ (e.g., $\mathcal{I}_{m} / \mathcal{N}_{0}>14 \mathrm{~dB}$ ), both cases obtain different performances. This comes from the fact that at large $\mathcal{I}_{m} / \mathcal{N}_{0}$, the transmit power depends on maximum transmit power levels and hence, the order of positioning unlicensed transmitters of different maximum transmit power levels considerably impact the BER of each hop, eventually varying the end-to-end BER. Moreover, Case 2 provides dramatically better performance than Case 1. This makes sense. According to simulation parameters, the fading powers are $\left[1 / \varrho_{01}, 1 / \varrho_{12}, 1 / \varrho_{23}\right]=[5.0441,89.4427,21.3346]$ for 


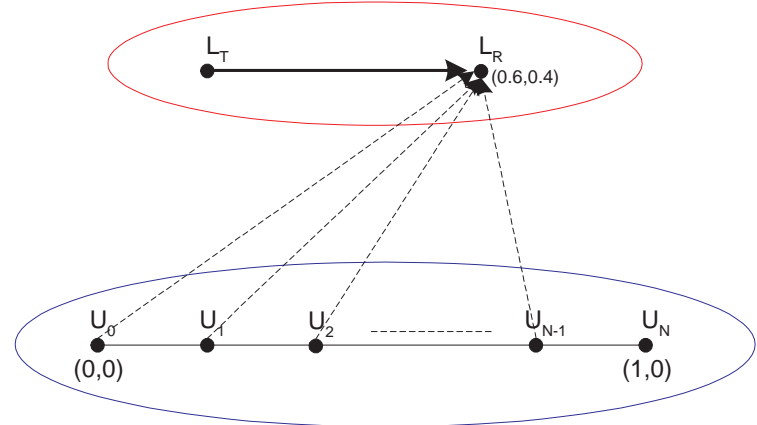

Figure 6. Linear network model.

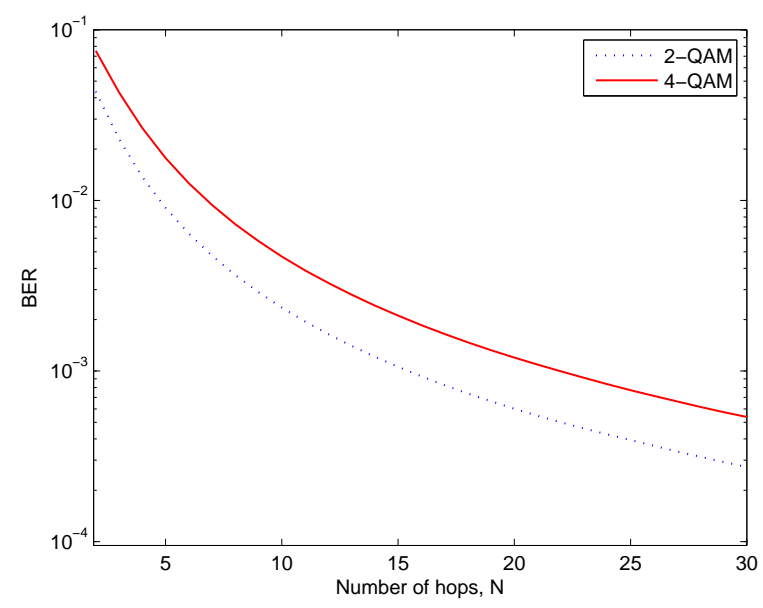

Figure 7. BER versus $N\left(\mathcal{P}_{m} / \mathcal{N}_{0}=40 \mathrm{~dB}, \mathcal{I}_{m} / \mathcal{N}_{0}=15 \mathrm{~dB}\right)$.

the 1st, 2nd, 3rd hop, respectively. Since the 1st hop suffers the most severe fading, the largest power should be allotted to this hop in order to guarantee the reliable information transmission over it, eventually resulting in the low end-to-end BER. As a result, Case 2 with the largest power for the 1st hop is more appropriate than Case 1 with the smallest power for it, and hence, Case 2 is better than Case 1 in terms of the end-to-end BER. Through this illustration, we recognize that as unlicensed transmitters have different maximum transmit power levels, their order in the multi-hop configuration becomes one of decisive factors drastically influencing the network performance. The topic on selecting the optimal order is interesting but outside the scope of the current paper.

The second part of this section investigates the performance behaviour versus the number of hops $N$. For this scenario, we consider the licensed user $L_{R}$ at $(0.6,0.4)$ and a linear network model in Figure 6 where $U_{0}$ and $U_{N}$ with $N=(2, \cdots, 30)$ are always located at $(0,0)$ and $(1,0)$, respectively while other relays (i.e., $U_{n}$ with $n \in\{1, \ldots, N-1\}$ ) are equally linear on the line connecting $U_{0}$ and $U_{N}$. For instance: only one relay is positioned at $(0.5,0)$ for two-hop communications, and two relays are located at $(1 / 3,0)$ and $(2 / 3,0)$ for threehop communications. This behaviour is clearly shown in Figure 7 for $\mathcal{I}_{m} / \mathcal{N}_{0}=15 \mathrm{~dB}, \mathcal{P}_{n m} / \mathcal{N}_{0}=\mathcal{P}_{m} / \mathcal{N}_{0}=$ $40 \mathrm{~dB}$ for all $n \in\{1, \ldots, N-1\},\{2-\mathrm{QAM}$ and 4-QAM $\}$

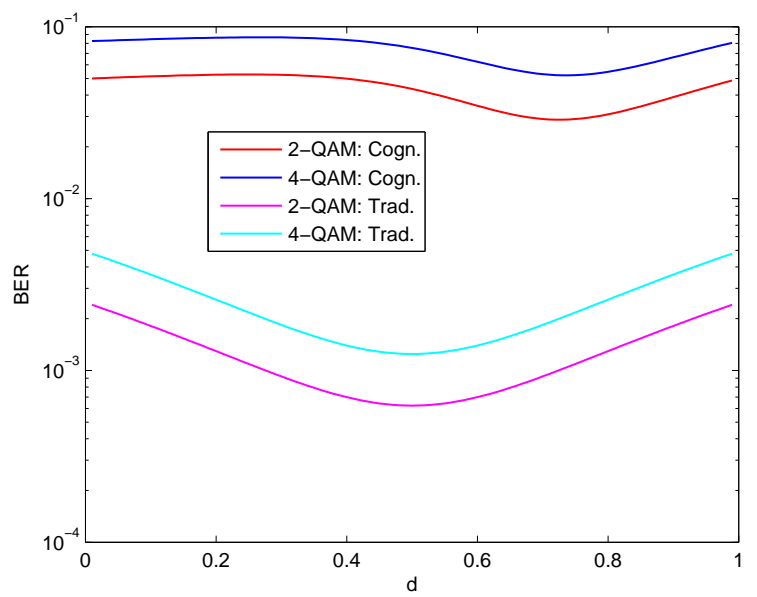

Figure 8. BER versus $U_{0}$-helper distance $\left(\mathcal{P}_{m} / \mathcal{N}_{0}=20 \mathrm{~dB}, \mathcal{I}_{m} / \mathcal{N}_{0}=\right.$ $10 \mathrm{~dB})$.

modulation, and $\tau=3$. As expected for the linear network topology, the higher number of hops reduces the path-loss component between two communication points. This ultimately constitutes the corresponding communication performance more reliable. Therefore, the results in Figure 7 are reasonable in the sense that the BER performance is significantly improved with the number of hops. Consequently, it is evident that in this type of linear network models, the underlay DF multihop cognitive network fully exploits the advantages of both multi-hop communications and cognitive radio for improving substantially the transmission range and spectrum efficiency.

It is recalled that the derived BER expressions are relative long but very convenient to handle both numerically and analytically. Therefore, they can provide several useful insights into the system performance without exhaustive and time-consuming simulations (see the above figures) as well as facilitate system design optimization. The third part of this section shows one of their system design optimization applications in finding the optimum relay position. For illustration purposes, we reconsider the linear network model with only one relay in Figure 6 . The source $U_{0}$ and the destination $U_{2}$ are located at $(0,0)$ and $(1,0)$ while the relay $U_{1}$ is at $(d, 0)$ with $0<d<1$. The problem is to find the optimum value of $d, d_{o p t}$, such that the BER is minimized i.e., $d_{o p t}=\min _{d} B_{e}$. The closed-form expression of $d_{o p t}$ is so involved but its value can be numerically computed using the derived BER expressions. Figure 8 with $\mathcal{P}_{m} / \mathcal{N}_{0}=20 \mathrm{~dB}, \mathcal{I}_{m} / \mathcal{N}_{0}=10 \mathrm{~dB}$, and the licensed user $L_{R}$ at $(0.6,0.4)$ demonstrates the BER of the underlay two-hop cognitive network labeled as 'Cogn.' with respect to $d$. As a reference, the results are also presented for the traditional two-hop network labeled as 'Trad.'. It is seen that the values of $d_{\text {opt }}$ are 0.73 and 0.5 for the two-hop cognitive network and the traditional two-hop networks, respectively. Also, they are independent of the modulation level, as expected. These solutions completely make sense. For the traditional two-hop network, it is obvious that the relay should be always located halfway $\left(d_{o p t}=0.5\right)$ between 


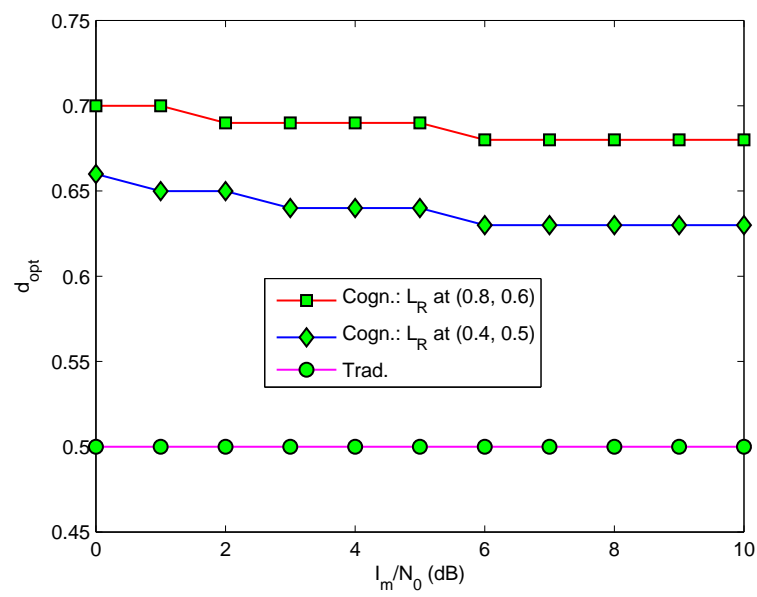

Figure 9. $d_{\text {opt }}$ versus $\mathcal{I}_{m} / \mathcal{N}_{0}\left(\mathcal{P}_{m} / \mathcal{N}_{0}=25 \mathrm{~dB}\right)$.

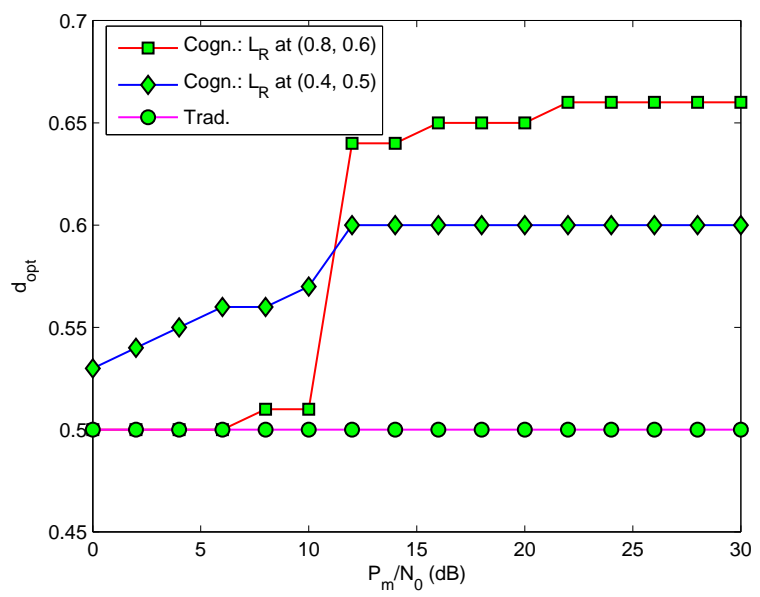

Figure 10. $d_{\text {opt }}$ versus $\mathcal{P}_{m} / \mathcal{N}_{0}\left(\mathcal{I}_{m} / \mathcal{N}_{0}=10 \mathrm{~dB}\right)$. the source $U_{0}$ and the destination $U_{2}$ for assisting equally both the source and the destination regardless of $\mathcal{P}_{m}$. Meanwhile, for the underlay two-hop cognitive network, the optimum relay position should not only improve the performance of the unlicensed network but also prevent to detrimentally interfere the licensed users. Therefore, the optimum relay position depends on many factors such as $\mathcal{I}_{m}$ and $\mathcal{P}_{m}$, and the licensed user's position. As a result, it can not be the same as that in the traditional two-hop network. Additionally, the performance of the traditional two-hop network is significantly better than that of the underlay two-hop cognitive network since the former upper-bounds the latter and the bound is tight as $\mathcal{I}_{m} \rightarrow \infty$, as discussed in Section 4. The two subsequent figures demonstrate results that justify the above remark addressing that the optimum relay position in the underlay two-hop cognitive network depends upon many factors such as $\mathcal{I}_{m}, \mathcal{P}_{m}$, the licensed user's position while that in the traditional two-hop network is always at $(0.5,0)$. Since the optimum relay position is independent of the modulation level, we only investigate the 2-QAM modulation. Figure 9 exposes $d_{\text {opt }}$ versus $\mathcal{I}_{m} / \mathcal{N}_{0}$ for $\mathcal{P}_{m} / \mathcal{N}_{0}=25 \mathrm{~dB}$ and two licensed user's positions at $(0.8,0.6)$ and $(0.4,0.5)$ while Figure 10 shows $d_{o p t}$ versus $\mathcal{P}_{m} / \mathcal{N}_{0}$ for $\mathcal{I}_{m} / \mathcal{N}_{0}=10 \mathrm{~dB}$. It is clearly shown that the above remark is fully validated.

\section{Conclusion}

This paper studied the BER analysis of underlay DF multi-hop cognitive networks in the presence of Rayleigh fading and under both interference power constraint and maximum transmit power constraint. Exact and asymptotic expressions were derived in closed-form and were verified with extensive computer simulations. Notably, asymptotic expressions can be efficiently used to compute the BER of underlay DF multi-hop cognitive networks under only interference power constraint and traditional DF multi-hop networks. The offered results demonstrate that underlay DF multi-hop cognitive networks ultimately incur the error floor due to two strict power constraints. Addi- tionally, as unlicensed users have different maximum transmit power levels, the appropriate order of locating them improves the network performance. Moreover, it is shown that for the linear network topology, the network performance increases significantly with increasing number of hops. Furthermore, the optimum relay position exists and depends on several factors such as maximum transmit power, maximum interference power, and the licensed user position.

\section{ACKNOWLEDGEMENT}

This research is funded by Vietnam National University HoChiMinh City (VNU-HCM) under grant number C2013-20-09.

\section{REFERENCES}

[1] FCC, "Spectrum policy task force report," ET Docket 02135, Nov. 2002.

[2] A. Goldsmith, S. A. Jafar, I. Maric, and S. Srinivasa, "Breaking spectrum gridlock with cognitive radios: An information theoretic perspective," Proceedings of the IEEE, vol. 97, no. 5, pp. 894-914, May 2009.

[3] J. Lee, H. Wang, J. G. Andrews, and D. Hong, "Outage probability of cognitive relay networks with interference constraints," IEEE Transactions on Wireless Communications, vol. 10, no. 2, pp. 390-395, Feb. 2011.

[4] J. N. Laneman, D. N. C. Tse, and G. W. Wornell, “Cooperative diversity in wireless networks: Efficient protocols and outage behavior," IEEE Transactions on Information Theory, vol. 50, no. 12, pp. 3062-3080, Dec. 2004.

[5] C. Zhong, T. Ratnarajah, , and K.-K. Wong, "Outage analysis of decode-and-forward cognitive dual-hop systems with the interference constraint in Nakagami-m fading channels," IEEE Transactions on Vehicular Technology, vol. 60, no. 6, pp. 2875-2879, June 2011.

[6] J. P. Hong, B. Hong, T. W. Ban, and W. Choi, "On the cooperative diversity gain in underlay cognitive radio systems," IEEE Transactions on Communications, vol. 60, no. 1, pp. 209-219, Jan. 2012.

[7] J. Si, H. H. Z. Li, J. Chen, and R. Gao, "Capacity analysis of cognitive relay networks with the PU's interference," IEEE Communications Letters, vol. 16, no. 12, pp. 20202023, Dec. 2012.

[8] W. Xu, J. Zhang, P. Zhan, and C. Tellambura, "Outage probability of decode-and-forward cognitive relay in presence of primary user's interference," IEEE Communications Letters, vol. 16, no. 8, pp. 1252-1255, Aug. 2012. 
[9] T. Q. Duong, P. L. Yeoh, V. N. Q. Bao, M. Elkashlan, and N. Yang, "Cognitive relay networks with multiple primary transceivers under spectrum-sharing," IEEE Signal Processing Letters, vol. 19, no. 11, pp. 741-744, Nov. 2012.

[10] J. Wang, Z. Zhang, Q. Wu, and Y. Huang, “Outage analysis of cognitive relay networks with interference constraints in Nakagami-m channels," IEEE Wireless Communications Letters, accepted.

[11] V. N. Q. Bao and T. Q. Duong, "Outage analysis of cognitive multihop networks under interference constraints," IEICE Transaction on Communications, vol. E95-B, no. 3, pp. 2019-1022, Mar. 2012.

[12] M. K. Simon and M. S. Alouini, Digital Communication over Fading Channels, 2nd ed. John Wiley and Son, 2005.

[13] Y. Guo, G. Kang, N. Zhang, W. Zhou, and P. Zhang, "Outage performance of relay-assisted cognitive-radio system under spectrum-sharing constraints," Electronics Letters, vol. 46, no. 2, pp. 182-184, Jan. 2010.

[14] Z. Yan, X. Zhang, and W. Wang, "Exact outage performance of cognitive relay networks with maximum transmit power limits," IEEE Communications Letters, vol. 15, no. 12, pp. 1317-1319, Dec. 2011.

[15] K. Ho-Van, "Exact outage probability of underlay cognitive cooperative networks over rayleigh fading channels," Wireless Personal Communications, vol. 70, no. 2, pp. 1001-1009, May 2013.

[16] L. Liping, P. Zhang, G. Zhang, and J. Qin, “Outage performance for cognitive relay networks with underlay spectrum sharing," IEEE Communications Letters, vol. 15, no. 7, pp. 710-712, July 2011.

[17] T. Q. Duong, D. B. da Costa, T. A. Tsiftsis, C. Zhong, and A. Nallanathan, "Outage and diversity of cognitive relaying systems under spectrum sharing environments in Nakagami-m fading," IEEE Communications Letters, vol. 16, no. 12, pp. 2075-2078, Dec. 2012.

[18] H. Ding, J. Ge, D. B. da Costa, and Z. Jiang, "Asymptotic analysis of cooperative diversity systems with relay selection in a spectrum-sharing scenario," IEEE Transactions on Vehicular Technology, vol. 50, no. 2, pp. 457-472, Feb. 2011.

[19] P. Yang, L. Liping, and Q. Jiayin, “Outage performance of cognitive relay networks with interference from primary user," IEEE Communications Letters, vol. 16, no. 10, pp. 1695-1698, Oct. 2012.

[20] Y. Zou, Y. D. Yao, and B. Zheng, "Cooperative relay techniques for cognitive radio systems: Spectrum sensing and secondary user transmissions," IEEE Communications Magazine, vol. 50, no. 4, pp. 98-103, Apr. 2012.

[21] V. N. Q. Bao and T. Q. Duong, "Exact outage probability of cognitive underlay DF relay networks with best relay selection," IEICE Transaction on Communications, vol. E95B, no. 6, pp. 2169-2173, June 2012.

[22] T. T. Duy and V. N. Q. Bao, "Outage performance of cooperative multihop transmission in cognitive underlay networks," in Proc. The International Conference on Computing, Management and Telecommunications, Hochiminh City, Vietnam, Jan. 2013, pp. 123-127.

[23] D. Li, "Cognitive relay networks: opportunistic or uncoded decode-and-forward relaying?" IEEE Transactions on Vehicular Technology, accepted.

[24] X. Zhang, Z. Yan, Y. Gao, and W. Wang, "On the study of outage performance for cognitive relay networks (CRN) with the Nth best-relay selection in Rayleigh-fading channels," IEEE Wireless Communications Letters, vol. 2, no. 1, pp. 110-113, Feb 2013.

[25] K. Tourki, K. A. Qaraqe, and M. S. Alouini, “Outage analysis for underlay cognitive networks using incremental regenerative relaying," IEEE Transactions on Vehicular Technology, vol. 62, no. 2, pp. 721-734, Feb 2013.

[26] H. Yu, W. Tang, and S. Li, "Outage probability and SER of amplify-and-forward cognitive relay networks," IEEE Wireless Communications Letters, vol. 2, no. 2, pp. 219-222, Apr. 2013.
[27] S. L. Hussain, M. M. Abdallah, M. S. Alouini, M. Hasna, and K. Qaraqe, "Performance analysis of selective cooperation in underlay cognitive networks over Rayleigh channels," in Proc. IEEE Inernational Workshop on Signal Processing Advances in Wireless Communications, San Francisco, California, USA, June 2011, pp. 116-120.

[28] T. Do and N. Mark, "Cooperative communication with regenerative relays for cognitive radio networks," in Proc. 44th Annual Conference in Information Sciences and Systems, Princeton, NJ, USA, Mar. 2010, pp. 1-6.

[29] K. Ho-Van and V. N. Q. Bao, "Symbol error rate of underlay cognitive relay systems over Rayleigh fading channel," IEICE Transaction on Communications, vol. E95B, no. 5, pp. 1873-1877, May 2012.

[30] E. Morgado, I. Mora-Jimenez, J. J. Vinagre, J. Ramos, and A. J. Caamano, "End-to-end average BER in multihop wireless networks over fading channels," IEEE Transactions on Wireless Communications, vol. 9, no. 8, pp. 24782487, Aug. 2010.

[31] N. Ahmed, M. Khojastepour, and B. Aazhang, "Outage minimization and optimal power control for the fading relay channel," in Proc. IEEE Information Theory Workshop, San Antonio, TX, USA, Oct. 2004, pp. 458-462.

[32] P. Athanasios and S. U. Pillai, Probability, Random Variables and Stochastic Process, 4th ed. McGraw Hill, 2002.

[33] K. Cho and D. Yoon, "On the general BER expression of one- and two-dimensional amplitude modulations," IEEE Transactions on Communications, vol. 50, no. 7, pp. 1074-1080, July 2002.

[34] I. S. Gradshteyn and I. M. Ryzhik, Table of Integrals, Series and Products, 6th ed. San Diego, CA: Academic Press, 2000.

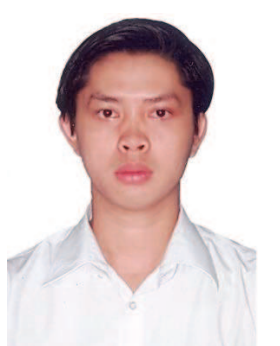

Khuong Ho-Van received the B.E. (with the first-rank honor) and the M.S. degrees in Electronics and Telecommunications Engineering from HoChiMinh City University of Technology, Vietnam, in 2001 and 2003, respectively, and the Ph.D. degree in Electrical Engineering from University of Ulsan, Korea in 2006. During 2007-2011, he joined McGill University, Canada as a postdoctoral fellow. Currently, he is an assistant professor at HoChiMinh City University of Technology. His major research interests are modulation and coding techniques, diversity technique, digital signal processing, and cognitive radio

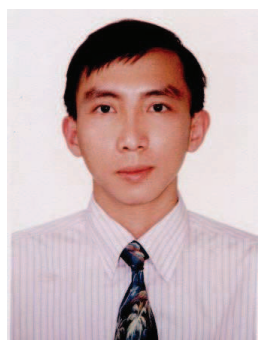

Vo Nguyen Quoc Bao was born in Nha Trang, Khanh Hoa Province, Vietnam. He received the B.E. and M.Eng. degree in electrical engineering from Ho Chi Minh City University of Technology (HCMUT), Vietnam, in 2002 and 2005, respectively, and $\mathrm{Ph} . \mathrm{D}$. degree in electrical engineering from University of Ulsan, South Korea, in 2009. In 2002, he joined the Department of Electrical Engineering, Posts and Telecommunications Institute of Technology (PTIT), as a lecturer. Since February 2010, he has been with the Department of Telecommunications, PTIT, where he is currently an Assistant Professor. His major research interests are modulation and coding techniques, MIMO systems, combining techniques, cooperative communications, and cognitive radio. Dr. Bao is a member of Korea Information and Communications Society (KICS), The Institute of Electronics, Information and Communication Engineers (IEICE) and The Institute of Electrical and Electronics Engineers (IEEE). He is currently serving as the Editor of Transactions on Emerging Telecommunications Technologies (Wiley ETT). He is also a Guest Editor of EURASIP Journal on Wireless Communications and Networking, special issue on "Cooperative Cognitive Networks" and IET Communications, special issue on "Secure Physical Layer Communications". 